\title{
Valoración de Empresa Transportadora en el Meta: Estudio de Caso
}

\author{
Valuation of a Transportation Company in the Meta: \\ Case Study
}

Como citar este artículo / To reference this articcle:

Perez-Diaz Kelly M. \& Romero-Herrera Yesica P., (2016). Valoración de Empresa Transportadora en el Meta: Estudio de Caso EL CONUCO: (investigación, economía y sociedad), 3(1), pp. 1-12. DOI: https://doi.org/10.22579/2619-614X.531

Kelly Melissa Pérez D. ${ }^{1}$

Yesica Paola Romero H. ${ }^{2}$

\section{Artículo de investigación}

Recepción: 08/15/2019

Aceptación: 12/18/2019

El Conuco es una revista de acceso abierto revisada por pares. (C) $2018 \mathrm{El}$ autor (es). Este es un artículo de acceso abierto distribuido bajo los términos de la Licencia Internacional Creative Commons Attribution 4.0 (CC-BY 4.0), que permite el uso, distribución y reproducción sin restricciones en cualquier medio, siempre que se acredite el autor y la fuente originales.

Consulte http://creativecommons.org/licenses/by/4.0/. ๑OPEN ACCESS

(c) $(1) \ominus$

1 Profesional en Salud Ocupacional, Contadora Pública, Especialista en Finanzas, Facultad de Ciencias Económicas, Universidad de los Llanos. Email: kelly_diaz01@hotmail. com, ORCID: https://orcid.org/0000-0001-5731-149X

2 Contadora Pública, Especialista en Finanzas, Facultad de Ciencias Económicas, Universidad de los Llanos. Email: yesicaromero.cp@gmail.com, ORCID: https://orcid. org/0000-0001-7098-3565

\section{Resumen}

El crecimiento de la economía ha sido el detonante de muchos cambios a nivel estructural y financiero de las empresas, en donde la búsqueda de creación de valor por parte de estas se convirtió en un objetivo principal y gracias a ello se le asignó un papel fundamental al área financiera dentro de las empresas siendo que "las finanzas buscaron la creación de valor, poniendo en funcionamiento nuevos esquemas de inversión, el apalancamiento financiero y operativo" (Crivellini, 2010, párr.3).

Por tal motivo se llega a la necesidad de la realización de la valoración por lo cual es necesario recurrir al histórico de tres años de Estados de Situación Financiera, que a su vez permitirá determinar la situación real de liquidez y los resultados operacionales de esta entidad, para aplicar el método de flujo de caja libre descontado, apto para empresas que no cotizan en bolsa y con el que se puede conocer la situación actual, si esta es favorable para exhibir ante inversionistas, futuros socios o compradores y si la gestión fue óptima.

Palabras Clave. Finanzas, Valoración de empresa, transporte, Flujo de caja libre descontado, Estados de situación financiera.

Códigos JEL: D21, G1, M10 


\section{Abstract}

The growth of the economy has been the trigger for many changes at the structural and financial level of the companies, where the search for the creation of value by them became a main objective and thanks to this, a fundamental role was assigned to the financial area within the companies being that "finances sought the creation of value, putting into operation new investment schemes, financial and operational leverage" (Crivellini, 2010, párr. 3).

For this reason, it is necessary to carry out the valuation for which it is necessary to resort to the three-year history of Financial Statements, which in turn will allow to determine the actual situation of liquidity and the operational results of this entity. , to apply the discounted free cash flow method, which is suitable for companies that are not listed on the stock exchange and with which you can know the current situation, if it is favorable to exhibit before investors, future partners or buyers and if the Management was optimal.

Keywords. Finance, Business Valuation, Transportation, Discounted Free Cash Flow, Financial Situation Statements.

\section{Introducción}

Las finanzas han demostrado ser un pilar fundamental para el crecimiento económico de las empresas, y la economía; mucho más por el auge de la globalización y la adopción de normas internacionales contables en Colombia, y la búsqueda de crecimiento, por lo tanto es importante incorporar en la empresa modelos de proyección financiera y medición de valor, aspectos que son desarrollados al realizar la valoración de una empresa, las empresas desconocen el valor real que tienen, por lo cual es necesario realizar una valoración, que permita determinar el valor económico de la empresa, y si es necesario implementar estrategias drásticas para incrementar el valor de la empresa o si la situación actual es favorable para exhibir ante inversionistas, futuros socios $\mathrm{O}$ compradores, con la certeza de dar un valor muy cercano a la realidad.

Así pues, existen diferentes modelos para valorar una empresa, entre los cuales se pueden encontrar el contable, basados en el Estado de situación financiera o en Múltiplos, o de Renta Futura tales como Valor de Mercado, Flujos de Caja Descontados y Opciones, sin embargo el más utilizado es el Flujo de Caja Descontados, que será el aplicado en el presente proyecto, teniendo en cuenta que la empresa no cotiza en bolsa y según los teóricos es el más óptimo para determinar el valor de una empresa.

La empresa objeto de estudio al cual se busca determinar el valor por medio del modelo previamente expuesto, es una empresa dedicada al servicio de transporte tanto público como privado y con clientes de prestigio internacional, para así definir este método como una herramienta de análisis y proyección de la misma.

\section{Metodología}

El crecimiento de la economía ha sido el detonante de muchos cambios a nivel estructural y financiero de las empresas, en donde la búsqueda de creación de valor por parte de estas se convirtió en un objetivo principal y gracias a ello se le asignó un papel fundamental al área financiera dentro de las empresas siendo que "las finanzas buscaron la creación de valor, poniendo en funcionamiento nuevos esquemas de inversión, el apalancamiento financiero y operativo" (Crivellini, 2010, Párr.3).

Así mismo, existen diferentes metodologías para apreciar dicha creación de valor, que se llamaron metodologías de valoración de empresa, dentro 
de las que se encuentra la teoría Flujo de Caja Libre Descontado, que se basa en tres elementos: Los Flujo de Caja Libre Operativos Proyectados, el Valor Terminal y la tasa de descuento, consecuentemente a lo que afirma Vidarte (2009). "Para valorar una empresa hay que tener en cuenta muchos factores y aplicar el método o los métodos que más convengan según el sector, el tamaño o el tipo la empresa que estemos valorando" (p. 104).

Así pues, el método del Flujo De Caja Descontado se basa en medir la capacidad de la empresa de generar riqueza futura, proyectando el flujo de caja libre (FCL), descontado a la tasa de descuento y restando los pasivos, o descontando el flujo de caja de los dueños (FCA) a su tasa de oportunidad (Vélez, 2001).

Teniendo en cuenta que el generador de valor es todo "Aspecto asociado con la operación del negocio que por tener relación causa- efecto con su valor, permite explicar el porqué de su aumento o disminución como consecuencia de las decisiones tomadas" (García, 2003, p.16).

El método de investigación, es el método inductivo, el cual parte de premisas particulares para producir un resultado especifico, dichas premisas son cuantitativas en lo que respecta a estados de Información financiera y lo cualitativas con base a las políticas contables y financieras de la empresa, lo que se aplica en el caso del objetivo principal deseado que es el establecimiento del valor de la empresa seleccionada el cual se logra mediante la aplicación de premisas previas como lo es la determinación de los flujo de caja libre operativos proyectados, el valor terminal y la tasa de descuento, basados en la información histórica de la empresa.

\section{Resultados}

Previamente sustraída la información contable y financiera de la empresa transportadora seleccio- nada ahora es posible iniciar la valoración de empresa, para empezar se realizará un diagnostico financiero de la empresa mediante análisis vertical que "permite determinar la participación de cada una de las cuentas del estado financiero, con referencia sobre el total de activos o total de pasivos y patrimonio para el balance general, o sobre el total de ventas para el estado de resultados" (Hevia, 2012, párr.1) y horizontal que "permite verificar la variación absoluta y la variación relativa que haya sufrido cada cuenta de un estado financiero con respecto de un periodo, a otro anterior" (Dussan, 2011, párr.1) y la aplicación de indicadores financieros, los cuales "Son utilizados para mostrar las relaciones que existen entre las diferentes cuentas de los estados financieros; y sirven para analizar su liquidez, solvencia, rentabilidad y eficiencia operativa de una entidad" (Alcántara, 2013, p.2).

Respecto a los generadores y destructores de valor de la empresa de transporte, podemos definir que "se crea valor cuando los administradores (de la empresa o del negocio), hacen las cosas bien; es decir, cuando las acciones tomadas generan una rentabilidad económica superior al costo de los recursos económicos o capital utilizados en el giro empresarial (Bonilla, 2010, p. 57). En la empresa objeto de valoración, se tiene como mayor generador de valor sus ventas, de las que se derivan alianzas y enlaza clientela incentivados por la calidad e innovación en el servicio, ya sea para carga, transporte público, transporte privado, en los cuales se invierte la mayor cantidad de tiempo y dinero, sin embargo va ligado al mayor destructor de valor que son los costos los cuales son muy volátiles y varían por diferentes condiciones externas no controlables al punto que pueden inferir drásticamente en los precios y a su vez en la demanda del servicio, así mismo la depreciación de los activos como la flota de transporte, por su continuo uso permiten el decremento de valor de los mismos. 
A partir del análisis del primer generador de valor, (volumen de ventas de la compañía y sus respectivos márgenes -bruto y operacional) se obtiene la generación operativa de fondos de la compañía (GOF), a la cual se le debe restar las inversiones de capital (en activos fijos y capital de trabajo) para obtener el flujo de caja libre operativo. Una vez se obtienen los flujos operativos proyectados se traen a valor presente con el costo promedio ponderado de capital (WACC) y así mismo obtener el valor de empresa según el análisis de escenarios (auge, optimista, normal, pesimista y crisis) para posteriormente establecer conclusiones y recomendaciones de acuerdo con el análisis de resultados.

\section{Diagnóstico Financiero}

En primer lugar en la realización del diagnóstico financiero se usa la información de los Estados Financieros históricos de la empresa de transportes seleccionada a los cuales se les aplicaran el análisis vertical y horizontal.

Posteriormente, se aplican una serie de indicadores financieros que permiten dictaminar el estado actual del ente económico y expresarlo en términos de fácil comprensión para cualquier usuario de esta información (véase tabla 1).

\section{Flujo de cala libre descontado}

La medición del flujo de caja libre descontado se basa en tres elementos: los flujos de caja libre proyectados, el valor terminal el cual como lo definen Tham \& Vélez (2012), es el valor de los flujos de caja que se generan en la firma o proyecto más allá del último período de la proyección. Se consideran situaciones con y sin crecimiento real y con y sin inflación y la tasa de descuento (WACC), con lo cual se obtiene un valor esperado de la empresa y los valores máximos y mínimos que podría negociar los socios de la empresa si desease vender, a continuación se muestra la metodología en la determinación de las herramientas para realizar el proceso.

\section{Costo del Patrimonio}

El cálculo se del costo del patrimonio se realiza a través del modelo de fijación de precios de activos de capital - CAMP, el cual se construye a

Tabla 1. Indicadores financieros

\begin{tabular}{|c|c|c|c|c|}
\hline Clase & Indicador & Año 2014 & Año 2015 & Año 2016 \\
\hline \multirow{4}{*}{ Indicadores de Liquidez } & Capital de trabajo & 1.112 .223 .598 & 1.329 .516 .944 & 1.340 .441 .279 \\
\hline & Liquidez corriente & 2,63 & 2,39 & 2,56 \\
\hline & Prueba Acida & 2,63 & 2,39 & 2,56 \\
\hline & Solvencia & 2,58 & 2,10 & 1,97 \\
\hline \multirow{4}{*}{ Endeudamiento o cobertura } & Endeudamiento del activo & $38,73 \%$ & $47,52 \%$ & $50,77 \%$ \\
\hline & Apalancamiento Total & $63,20 \%$ & $90,55 \%$ & $103,12 \%$ \\
\hline & Apalancamiento Financiero & $22,17 \%$ & $56,34 \%$ & $76,89 \%$ \\
\hline & Concentración del pasivo & $80,91 \%$ & $63,57 \%$ & $50,29 \%$ \\
\hline \multirow{9}{*}{ Rentabilidad o Rendimiento } & ROA Rentabilidad sobre Activos & $14,43 \%$ & $20,96 \%$ & $24,35 \%$ \\
\hline & Margen Operativo & $10,81 \%$ & $13,51 \%$ & $12,15 \%$ \\
\hline & Margen Neto & $5,26 \%$ & $6,64 \%$ & $5,03 \%$ \\
\hline & Carga Administrativa & $21,82 \%$ & $14,66 \%$ & $5,95 \%$ \\
\hline & Carga Operativa & $22,52 \%$ & $14,66 \%$ & $5,95 \%$ \\
\hline & Carga Financiera & $4,18 \%$ & $1,67 \%$ & $3,61 \%$ \\
\hline & Sistema DUPONT & $1,56 \%$ & $5,80 \%$ & $7,75 \%$ \\
\hline & ROE Rentabilidad Patrimonio & $11,46 \%$ & $19,63 \%$ & $20,49 \%$ \\
\hline & Ebitda & $594.494 .847,00$ & $973.514 .406,00$ & $822.007 .995,00$ \\
\hline
\end{tabular}

Nota: Elaboración propia con los resultados de los indicadores financieros a la información contable de los respectivos años. 
partir de información macroeconómica de entes especializados a nivel mundial como, Damodaran, JP Morgan Chase, el Banco de la Republica de Colombia (s.f), entre otros. La fórmula aplicada del modelo, KJ: KF + $(\mathrm{KM}-\mathrm{KF}) * \mathrm{~b}+\mathrm{RP}$, donde, KF es la Tasa de libre de riesgo, KM es la Rentabilidad del mercado, b es Beta Apalancada, y RP es Riesgo país.

Tabla 2. Calculo del CAMP

\begin{tabular}{lc}
\hline CAMP & $\mathbf{1 1 , 1 0 \%}$ \\
\hline $\mathbf{( + )}$ EMBI & $\mathbf{2 , 2 5 \%}$ \\
\hline CAMP AJUSTADO & $\mathbf{1 3 , 3 5 \%}$ \\
\hline Tess- Tasa LR & $6,62 \%$ \\
\hline Rentabilidad sector & $9,80 \%$ \\
\hline Beta & 1,41 \\
\hline EMBI & $\mathbf{2 , 2 5 \%}$ \\
\hline
\end{tabular}

Nota: Elaboración propia con los datos recolectados para el cálculo del CAMP

\section{Determinación del WACC}

Por lo tanto, el Costo Promedio Ponderado de Capital - WACC, se puede definir como la tasa mínima de retorno que induzca a los inversionistas a invertir en una empresa, con el fin de de- terminar el costo promedio ponderado de capital de la empresa, tras realizar un análisis preliminar para hallar el costo de cada una de las obligaciones financieras, que son aquellos costos para desarrollar su actividad o un proyecto de inversión a través de su financiación en forma de créditos y préstamos o emisión de deuda tal como lo afirma Velásquez (2012), teniendo así catorce obligaciones para la empresa estudio hasta el 2016.

De la misma forma como lo define Ortiz (2015) "Es el costo que tienen para la empresa las diferentes fuentes de financiación tales como endeudamiento financiero, patrimonio y proveedores. Esta tasa es bastante importante ya que es la que determina de forma exacta el costo mínimo de los recursos" (p.7).

Por lo tanto, una vez se obtienen los flujos operativos proyectados se traen a valor presente con el costo promedio ponderado de capital (WACC) y así mismo obtener el valor de empresa según el análisis de escenarios.

Tabla 3. WACC

\begin{tabular}{llccc}
\hline \multicolumn{1}{c}{ Fuente } & Valor Fuente & Costo Fuente & Par. Partic & WACC \\
\hline Crédito Bogotá 09451011352 & $\$ 110.987 .949$ & $10,68 \%$ & $3,7973 \%$ & $0,4055 \%$ \\
\hline Tarjeta de Credito 4704350001465178 & $\$ 110.407$ & $21,71 \%$ & $0,0038 \%$ & $0,0008 \%$ \\
\hline Tarjeta de Credito SKV-008 ${ }^{* * *} 6148$ & $\$ 251.211$ & $21,71 \%$ & $0,0086 \%$ & $0,0019 \%$ \\
\hline Tarjeta de Credito SJK-099 ${ }^{* * *} 6113$ & $\$ 1.724 .128$ & $21,71 \%$ & $0,0590 \%$ & $0,0128 \%$ \\
\hline Tarjeta de Credito TKG-875 ${ }^{* * *} 0262$ & $\$ 377.785$ & $21,71 \%$ & $0,0129 \%$ & $0,0028 \%$ \\
\hline Crédito Bogotá 259033940 & $\$ 19.288 .890$ & $10,29 \%$ & $0,6599 \%$ & $0,0679 \%$ \\
\hline Banco BBVA \# 957-1000-14734 & $\$ 191.878 .600$ & $10,74 \%$ & $6,5649 \%$ & $0,7050 \%$ \\
\hline Banco BBVA \# 957-1000-14740 & $\$ 191.878 .600$ & $10,74 \%$ & $6,5649 \%$ & $0,7050 \%$ \\
\hline Banco de Bogotá \# 259316617 & $\$ 215.677 .096$ & $11,80 \%$ & $7,3792 \%$ & $0,8708 \%$ \\
\hline Davivienda \# 0001000579 & $\$ 356.923 .431$ & $12,06 \%$ & $12,2118 \%$ & $1,4733 \%$ \\
\hline Banco de Occidente Credito 710-0005148-3 & $\$ 68.888 .890$ & $11,08 \%$ & $2,3570 \%$ & $0,2612 \%$ \\
\hline Credito Bco Bta 353389004 & $\$ 2.495 .726$ & $8,54 \%$ & $0,0854 \%$ & $0,0073 \%$ \\
\hline Credito Occidente No 71000051895 & $\$ 66.916 .666$ & $11,08 \%$ & $2,2895 \%$ & $0,2537 \%$ \\
\hline Banco Ocidente Prestamo $* * * 5152-7$ & $\$ 43.055 .555$ & $11,07 \%$ & $1,4731 \%$ & $0,1630 \%$ \\
\hline Patrimonio & $\$ 1.652 .325 .978$ & $13,35 \%$ & $56,5327 \%$ & $7,5493 \%$ \\
\hline TOTAL & $\$ 2.922 .780 .912$ & & $100,00 \%$ & $12,48 \%$ \\
\hline Not
\end{tabular}

Nota: Elaboración propia con base a la información de las obligaciones financieras fue suministrada por la empresa objeto de estudio, donde se expresa el valor de cada obligación y su respectiva tasa de interés. 


\section{Valoración de Empresa}

El primer paso para realizar la valoración de la empresa objeto de estudio, es la elaboración del flujo de caja libre descontado en cada escenario.

Así pues, se presenta a continuación el flujo de caja para el escenario normal resultante del ejercicio de proyección (ver tabla 4):

Cabe destacar que, en relación a la actividad económica desarrollada por la empresa objeto de estudio, el incremento de sus ingresos se fundamenta en la flota de transporte apta y suficiente para cumplir con su demanda, además de considerar la vida útil de la misma en constante uso; es por ello que se establece como parámetro una reserva con destinación específica de sus utilidades del 40\% para los dos primeros años y del $30 \%$ para los siguientes años, que tendrá como objetivo la compra de los activos previamente mencionados para los años 2019 y 2021.
De igual forma, se parte desde una utilidad operacional, la cual para efectos de determinar el flujo de caja bruto se le restan los impuestos resultantes del ejercicio, y se le suman las depreciaciones; luego con el fin de obtener el flujo de caja libre operativo, se restan la inversión en activos fijos que es necesaria para la continuidad de la empresa, y los abonos a capital de los créditos e la empresa, lo cual afecta en una proporción considerable el flujo de la empresa.

El valor que aparece como nuevo préstamo en el primer año, aparece de una modalidad de apalancamiento con deuda que maneja la empresa; para efectos de la valoración se amortiza en el primer año, tomando su valor promedio para los cinco años que se estima la operación de la empresa.

La variación de KTNO se determinó realizando el cálculo con las cifras proyectadas en el Estado de Situación Financiera obteniendo lo siguiente: (ver tabla 5)

Tabla 4. Flujo de Caja Libre, escenario normal

\begin{tabular}{llcccc}
\hline \multicolumn{7}{c}{ Flujo de Caja Libre Proyectado } & & \\
\hline & \multicolumn{1}{c}{$\mathbf{2 0 1 7}$} & $\mathbf{2 0 1 8}$ & $\mathbf{2 0 1 9}$ & $\mathbf{2 0 2 0}$ & $\mathbf{2 0 2 1}$ \\
\hline U. Operacional & $\$ 1.226 .941 .167$ & $\$ 1.614 .374 .575$ & $\$ 1.972 .349 .774$ & $\$ 2.206 .472 .197$ & $\$ 2.548 .501 .344$ \\
\hline$(-)$ impuestos & $\$(365.564 .371)$ & $\$(500.393 .096)$ & $\$(635.028 .881)$ & $\$(727.768 .318)$ & $\$(850.653 .700)$ \\
\hline$(+)$ Depreciaciones & $\$ 232.102 .173$ & $\$ 202.788 .579$ & $\$ 202.079 .300$ & $\$ 324.387 .137$ & $\$ 337.902 .114$ \\
\hline (=)Flujo de Caja Bruto & $\$ 1.093 .478 .969$ & $\$ 1.316 .770 .058$ & $\$ 1.539 .400 .193$ & $\$ 1.803 .091 .016$ & $\$ 2.035 .749 .758$ \\
\hline$(-)$ Inversion en activos fijos & & & $\$(690.229 .830)$ & & $\$(830.067 .385)$ \\
\hline$(-)$ Abono creditos & $\$(415.439 .937)$ & $\$(351.843 .363)$ & $\$(349.292 .558)$ & $\$(343.425 .007)$ & $\$(283.332 .473)$ \\
\hline$(-)$ Variacion KTNO & $\$-$ & $\$(358.753 .584)$ & $\$(327.739 .855)$ & $\$(296.921 .253)$ & $\$(305.418 .499)$ \\
\hline$(+)$ Nuevos Creditos & $\$ 516.000 .000$ & & & & \\
\hline (=) Flujo de Caja Libre & $\$ 1.194 .039 .032$ & $\$ \mathbf{6 0 6 . 1 7 3 . 1 1 1}$ & $\$ 172.137 .951$ & $\$ 1.162 .744 .756$ & $\$ 616.931 .402$ \\
\hline
\end{tabular}

Nofa: Elaboración propia con base a la información financieras proyectada con las variables económicas consideradas para un escenario normal con base al método de mínimo cuadrados, a los promedios de participación, el incremento del SMMLV y la inflación para el año 2016.

Tabla 5. KTNO, escenario normal

\begin{tabular}{|c|c|c|c|c|c|c|c|c|c|}
\hline \multicolumn{10}{|c|}{ Variacion del Capital de Trabajo Neto Opeativo } \\
\hline & & 2017 & & 2018 & & 2019 & & 2020 & 2021 \\
\hline Cuentas Comerciales Por Cobrar & $\$$ & 1.974 .041 .906 & $\$$ & 2.375 .338 .082 & $\$$ & 2.745 .722 .997 & $\$$ & 3.085 .389 .590 & $\$ 3.436 .234 .208$ \\
\hline Cuentas Comerciales Por Pagar & $\$$ & 213.559 .781 & $\$$ & 256.102 .373 & $\$$ & 298.747 .433 & $\$$ & 341.492 .772 & $\$ 386.918 .892$ \\
\hline KTNO & $\$$ & 1.760 .482 .125 & $\$$ & 2.119 .235 .709 & $\$$ & 2.446 .975 .565 & $\$$ & 2.743 .896 .818 & $\$ 3.049 .315 .317$ \\
\hline Variacion KTNO & & & $\$$ & 358.753 .584 & $\$$ & 327.739 .855 & $\$$ & 296.921 .253 & $\$ 305.418 .499$ \\
\hline
\end{tabular}

Nota: Elaboración propia con base a la información financieras de la empresa proyecta dentro del escenario normal con base a las variables económicas previamente expuestas. 
Previo a la determinación del flujo d caja libre proyectado y el costo promedio ponderado de capital- WACC se procede a estimar el valor de la empresa, mediante el proceso de valor terminal o perpetuo, el cual consta de cinco pasos.

\section{Valor de Empresa Escenario Normal}

Para poder determinar el valor de empresa en los respectivos escenarios se debe tener en cuenta lo siguiente:

Inicialmente se debe hallar el valor presente neto de dicho flujo mediante la aplicación del WACC, como tasa de descuento.

Seguidamente se proyecta el último Flujo de Caja Explicito al siguiente periodo; y se calcula el Valor Perpetuo del flujo de caja seis (6) en adelante, el cual se halla mediante la aplicación de la fórmula VT: FCLn+1 / (WACC-G), donde FCLn+1 es el flujo de caja libre del último periodo de proyección, y $G$ es el gradiente de crecimiento que corresponde a la tasa de crecimiento fundamental del ROE para la empresa que en este caso es la tasa de crecimiento del sector transporte presentada por las estadísticas del estudio del sector transporte. De tal forma que el valor de la empresa es la suma entre el valor presente del periodo explícito de proyección y el valor presente del periodo de continuidad. A continuación se presentan la estimación del valor de la empresa:

Tabla 6. Valor de Empresa, escenario normal

\begin{tabular}{rlc}
\hline \multicolumn{3}{c}{ Valoración de Empresa en Escenario Normal } \\
\hline 1. & Valor Presente Flujo de Caja $=$ & $\$ 2.730 .695 .617$ \\
\hline 2. & FC Normalizado $=$ & $\$ 624.951 .511$ \\
\hline & Valor Perpetuo $=$ & FC $/($ WACC-g $)$ \\
\hline 3. & Valor Perpetuo $=$ & $\$ 5.589 .782 .669$ \\
\hline & Valor Presente $=$ & FC $/(1-1) \wedge n$ \\
\hline 4. & VPN $=$ & $\$ 3.104 .656 .920$ \\
\hline 5. & Valor de la Empresa $=$ & $\$ 5.835 .352 .537$ \\
\hline
\end{tabular}

Nota: Elaboración propia con base a la información obtenida del flujo de caja libre en un escenario normal
El valor de la empresa objeto de estudio bajo las proyecciones normales propuestas es de $\$ 5.835 .352 .537$ el cual es un valor razonable comparándolo con el valor en libros de la empresa estimado por su activo, y teniendo en cuenta la generación de valor por medio de los flujos de caja de la empresa; y la rentabilidad esperada.

\section{Análisis de sensibilidad}

Debido a que existen un grado de incertidumbre en las estimaciones de proyección, y que dicha incertidumbre incide directamente en el valor de la empresa, se procede a realizar la valoración bajo dos escenarios adicionales al escenario normal, igualmente con las cifras anteriormente proyectadas para cada escenario; a continuación se proyectan los valores resultantes de los escenarios: optimista y pesimista, con los cuales se busca identificar el valor de la empresa objeto de estudio, con el objetivo de buscar el menor grado de incertidumbre posible ante las variaciones de las estimaciones en unas condiciones dadas por el comportamiento histórico del mercado.

\section{Valor de Empresa Escenario Optimista}

Las variables estimadas para este escenario fueron anteriormente proyectadas y parametrizadas, a continuación se muestra el Flujo de Caja Libre y el proceso para determinar el valor de la empresa bajo este escenario: (véase tabla 7 )

Como había sido previamente mencionado, es importante mantener una adecuada flota de transporte, debido a que de esto depende el flujo de ingresos, es por lo que en este caso se establece igual que en las condiciones como parámetro y de acuerdo con los ingresos una reserva de sus utilidades del 45\% para los dos primeros años y del $35 \%$ para los siguientes años, que será utilizada en la compra de los activos en el año 2019 y 2021. 
Tabla 7. Flujo de Caja Libre, escenario optimista

\begin{tabular}{|c|c|c|c|c|c|c|c|c|c|c|}
\hline \multicolumn{11}{|c|}{ Flujo de Caja Libre Proyectado } \\
\hline & & 2017 & & 2018 & & 2019 & & 2020 & & 2021 \\
\hline U. Operacional & & 1.295 .434 .503 & $\$$ & 1.927 .490 .524 & $\$$ & 2.754 .754 .857 & $\$$ & 3.725 .092 .719 & $\$$ & 5.127 .852 .572 \\
\hline (-)impuestos & $\$$ & $(388.852 .105)$ & $\$$ & $(603.721 .359)$ & $\$$ & $(893.222 .558)$ & $\$$ & $(1.228 .913 .090)$ & $\$$ & $(1.701 .839 .605)$ \\
\hline$(+)$ Depreciaciones & $\$$ & 232.102 .173 & $\$$ & 202.788 .579 & $\$$ & 202.079 .300 & $\$$ & 364.592 .279 & $\$$ & 513.694 .269 \\
\hline (=)Flujo de Caja Bruto & $\$$ & 1.138 .684 .571 & $\$$ & 1.526 .557 .744 & $\$$ & 2.063 .611 .599 & $\$$ & 2.860 .771 .908 & $\$$ & 3.939 .707 .236 \\
\hline (-) Inversion en activos fijos & & & & & $\$$ & $(891.255 .538)$ & & & $\$$ & $(1.508 .002 .453)$ \\
\hline (-) Abono creditos & $\$$ & $(415.439 .937)$ & $\$$ & $(351.843 .363)$ & $\$$ & $(349.292 .558)$ & $\$$ & $(343.425 .007)$ & $\$$ & $(283.332 .473)$ \\
\hline (-)Variacion KTNO & & & $\$$ & (555.370.795) & $\$$ & $(687.790 .199)$ & $\$$ & $(873.237 .818)$ & $\$$ & $(1.189 .775 .244)$ \\
\hline (+) Nuevos Creditos & $\$$ & 516.000 .000 & & & & & & & & \\
\hline$(=)$ Flujo de Caja Libre & $\$$ & 1.239 .244 .634 & $\$$ & 619.343 .586 & $\$$ & 135.273.304 & $\$$ & 1.644 .109 .083 & $\$$ & 958.597 .066 \\
\hline
\end{tabular}

Nota: Elaboración propia con base a la información financieras proyectada con las variables económicas consideradas para un escenario optimista con base a los promedios de participación, el incremento del SMMLV para el año 2016

De igual forma se parte desde una utilidad operacional, a la cual para efectos de determinar el flujo de caja bruto se le restan los impuestos resultantes del ejercicio, y se le suman las depreciaciones; luego con el fin de obtener el flujo de caja libre operativo se restan la inversión en activos fijos necesarios para la continuidad de la empresa, y los abonos a capital de los créditos de la empresa, lo cual afecta en una participación considerable el flujo de la empresa.
La variación de KTNO se determinó realizando el cálculo con las cifras proyectadas en el Estado de Situación Financiera obteniendo lo siguiente: (véase tabla 8)

Previo a la determinación del flujo de caja libre proyectada y el costo promedio ponderado de capital- WACC que es el mismo del escenario normal, se procede a estimar el valor de la empresa desde el escenario optimista, mediante el proceso de valor terminal o perpetuo igualmente.

Tabla 8. KTNO, escenario optimista

\begin{tabular}{llllll}
\hline \multicolumn{5}{c}{ Variacion del Capital de Trabajo Neto Opeativo } \\
\hline Cuentas Comerciales Por Cobrar & $\$ 2.056 .224 .840$ & $\$ 2.690 .865 .784$ & $\$ 3.486 .783 .105$ & $\$ 4.507 .582 .763$ & $\$ 5.896 .117 .375$ \\
\hline Cuentas Comerciales Por Pagar & $\$ 358.197 .449$ & $\$ 437.467 .598$ & $\$ 545.594 .720$ & $\$ 693.156 .561$ & $\$ 891.915 .929$ \\
\hline KTNO & $\$ 1.698 .027 .391$ & $\$ 2.253 .398 .186$ & $\$ 2.941 .188 .385$ & $\$ 3.814 .426 .203$ & $\$ 5.004 .201 .447$ \\
\hline Variacion KTNO & & $\$ 555.370 .795$ & $\$ 687.790 .199$ & $\$ 873.237 .818$ & $\$ 1.189 .775 .244$ \\
\hline
\end{tabular}

Nota: Elaboración propia con base a la información financieras de la empresa proyecta dentro del escenario optimista con base a las variables económicas previamente expuestas.

Tabla 9. Valor de Empresa, escenario optimista

\begin{tabular}{llc}
\hline & \multicolumn{2}{l}{ Valoración de Empresa en Escenario Normal } \\
\hline 1. & Valor Presente Flujo de Caja $=$ & $\$ 3.245 .881 .767$ \\
\hline 2. & FC Normalizado $=$ & $\$ 971.058 .828$ \\
\hline & Valor Perpetuo $=$ & FC $/($ WACC-g $)$ \\
\hline 3. & Valor Perpetuo $=$ & $\$ 8.685 .486 .338$ \\
\hline & Valor Presente $=$ & FC $/(1-\mathrm{i})^{\wedge} \mathrm{n}$ \\
\hline 4. & VPN $=$ & $\$ 4.824 .061 .481$ \\
\hline 5. & Valor de la Empresa $=$ & $\$ 8.069 .943 .248$ \\
\hline
\end{tabular}

Nota: Elaboración propia con base a la información obtenida del flujo de caja libre en un escenario optimista
El valor de la empresa objeto de estudio bajo las proyecciones Optimistas propuestas es de $\$ 8.069 .943 .248$, el cual es un valor estimado que aunque se aleja de las proyecciones normales, responde al crecimiento y la generación de valor por medio de los flujos de caja de la empresa en este escenario. 


\section{Valor de Empresa Escenario Pesimista}

Las proyecciones para el escenario pesimista se desarrollaron con base al diagnóstico financiero, las tendencias aplicables de la economía del País, a través de una combinación de variables microeconómicas y macroeconómicas.

Así mismo, las variables estimadas para este escenario fueron anteriormente proyectadas y parametrizadas, a continuación se muestra el Flujo de Caja Libre y el proceso para determinar el valor de la empresa bajo este escenario: (véase tabla 10

En este escenario la reserva de sus utilidades será del 35\% para los dos primeros años y del 25\% para los siguientes años, la cual será utilizada en la compra de los activos en el año 2019 y 2021 respectivamente.

De igual forma se parte desde una utilidad operacional, a la cual para efectos de determinar el flujo de caja bruto se le restan los impuestos resultantes del ejercicio, y se le suman las depreciaciones; luego con el fin de obtener el Flujo de Caja Libre Operativo se restan la inversión en activos fijos que es necesaria para la continuidad de la empresa, y los abonos a capital de los créditos e la empresa, lo cual afecta en una participación considerable el flujo de la empresa.

La variación de KTNO se determinó realizando el cálculo con las cifras proyectadas en el Estado de Situación Financiera obteniendo lo siguiente: (véase tabla 11)

Previo a la determinación del flujo de caja libre proyectada y el costo promedio ponderado de capital- WACC que es el mismo del escenario normal, se procede a estimar el valor de la empresa en un escenario pesimista, mediante el proceso de valor terminal o perpetuo igualmente.

Tabla 10. Flujo de Caja Libre, escenario pesimista

\begin{tabular}{|c|c|c|c|c|c|c|c|}
\hline \multicolumn{8}{|c|}{ Flujo de Caja Libre Proyectado } \\
\hline & & 2017 & & 2018 & 2019 & 2020 & 2021 \\
\hline U. Operacional & $\$$ & 986.238 .893 & $\$$ & 1.153 .776 .976 & $\$ 1.307 .722 .442$ & $\$ 1.383 .462 .573$ & $\$ 1.640 .471 .112$ \\
\hline (-)impuestos & $\$$ & $(262.512 .495)$ & $\$$ & $(323.276 .280)$ & $\$(386.051 .833)$ & $\$(421.994 .693)$ & $\$(512.292 .855)$ \\
\hline (+) Depreciaciones & $\$$ & 232.102 .173 & & 202.788 .579 & $\$ 202.079 .300$ & \$ 296.266 .412 & $\$ 227.660 .748$ \\
\hline$(=)$ Flujo de Caja Bruto & $\$$ & 955.828 .572 & $\$$ & 1.033.289.275 & $\$ 1.123 .749 .909$ & $\$ 1.257 .734 .292$ & $\$ 1.355 .839 .005$ \\
\hline (-) Inversion en activos fijos & & & & & $\$(408.076 .161)$ & & $\$(410.144 .828)$ \\
\hline (-) Abono creditos & $\$$ & $(415.439 .937)$ & $\$$ & $(351.843 .363)$ & $\$(349.292 .558)$ & $\$(343.425 .007)$ & $\$(283.332 .473)$ \\
\hline (-)Variacion KTNO & $\$$ & & $\$$ & $(244.297 .514)$ & $\$(236.661 .489)$ & $\$(232.589 .087)$ & $\$(259.198 .462)$ \\
\hline (+) Nuevos Creditos & $\$$ & 516.000 .000 & & & & & \\
\hline$(=$ Flujo de Caja Libre & $\$$ & 1.056 .388 .634 & $\$$ & 437.148 .398 & $\$ 129.719 .702$ & \$ 681.720 .198 & $\$ 403.163 .242$ \\
\hline
\end{tabular}

Nota: Elaboración propia con base a la información financieras proyectada con las variables económicas consideradas para un escenario pesimista con base a los promedios de participación, el incremento de la tasa de inflación para el año 2016

Tabla 11. KTNO, escenario pesimista

\begin{tabular}{lllllll}
\hline \multicolumn{7}{c}{ Variación del Capital de Trabajo Neto Opeativo } \\
\hline Centas Comerciales Por Cobrar & $\$ 1.783 .133 .641$ & $\$ 2.045 .733 .005$ & $\$ 2.302 .547 .797$ & $\$ 2.557 .322 .570$ & $\$ 2.843 .249 .029$ \\
\hline Centas Comerciales Por Pagar & $\$ 187.025 .330$ & $\$ 205.327 .180$ & $\$ 225.480 .483$ & $\$ 247.666 .170$ & $\$ 274.394 .166$ \\
\hline KTNO & $\$ 1.596 .108 .311$ & $\$ 1.840 .405 .825$ & $\$ 2.077 .067 .313$ & $\$ 2.309 .656 .401$ & $\$ 2.568 .854 .863$ \\
\hline Variacion KTNO & & $\$ 244.297 .514$ & $\$ 236.661 .489$ & $\$ 232.589 .087$ & $\$ 259.198 .462$ \\
\hline
\end{tabular}

Noła: Elaboración propia con base a la información financiera de la empresa proyecta dentro del escenario pesimista con base a las variables económicas previamente expuestas. 
Tabla 12. Valor de Empresa, escenario pesimista

\begin{tabular}{clc}
\hline \multicolumn{3}{c}{ Valoración de Empresa en Escenario Normal } \\
\hline 1. & Valor Presente Flujo de Caja $=$ & $\$ 2.025 .670 .924$ \\
\hline 2. & FC Normalizado $=$ & $\$ 408.404 .365$ \\
\hline & Valor Perpetuo $=$ & FC $/($ WACC-g $)$ \\
\hline 3. & Valor Perpetuo $=$ & $\$ 3.652 .910 .025$ \\
\hline & Valor Presente $=$ & FC $/(1-\mathrm{i})^{\wedge} \mathrm{n}$ \\
\hline 4. & VPN $=$ & $\$ 2.028 .886 .105$ \\
\hline 5. & Valor de la Empresa $=$ & $\$ 4.054 .557 .029$ \\
\hline
\end{tabular}

Nota: Elaboración propia con base a la información obtenida del flujo de caja libre en un escenario pesimista

El valor de la empresa objeto de estudio bajo las proyecciones Pesimistas propuestas es de \$4.054.557.029, el cual es un valor estimado que obedece a las variables propuestas en unas condiciones de mercado de bajo crecimiento y generación de valor.

\section{Valor de Negociación de la empresa.}

Los escenarios están basados en las proyecciones realizadas anteriormente, cada escenario está determinado por valores estimados dados por las variables alteradas para cada situación. Normalmente se definen tres escenarios: optimista, normal y pesimista; cada escenario nos permite examinar los resultados cuando alteramos las variables, midiendo sus cambios tanto porcentual como relativamente, y de esa forma encontrar un valor de empresa, contemplando diferentes posibilidades del comportamiento económico del ente.

La medición del Flujo de Caja Libre Descontado se basa en tres elementos: Los Flujos de Caja Libre Proyectados, el Valor Terminal y la tasa de descuento (WACC), con lo cual se obtiene un valor esperado de la empresa y los valores máximos y mínimos que podría negociar los socios de la empresa si desease vender, a continuación se muestra la metodología en la determinación de las herramientas para realizar el proceso.

De acuerdo con el análisis de sensibilidad se halló el valor esperado de la empresa y su valor o precio base de negociación, el cual representa el valor que más se acerca al valor real de la compañía y el monto mínimo por el que se debería negociar la empresa, esté se obtiene sumando los valores resultantes de la multiplicación entre la probabilidad de ocurrencia de cada escenario por el valor de la empresa obtenido en ellos, menos la desviación estándar.

Tabla 13. Matriz de Resultados de los Escenarios

\begin{tabular}{lcc}
\hline \multicolumn{3}{c}{ Matriz De Resultados } \\
\hline Escenarios & Valor de la Empresa & Probabilidad \\
\hline Escenario optimista & $\$ 8.069 .943 .248$ & $25 \%$ \\
\hline Escenario normal & $\$ 5.835 .352 .537$ & $50 \%$ \\
\hline Escenario pesimista & $\$ 4.054 .557 .029$ & $25 \%$ \\
\hline
\end{tabular}

Nota: Elaboración propia con base a los resultados de valor de empresa en cada uno de los escenarios previamente expuestos.

Los escenarios están basados en las proyecciones realizadas anteriormente, cada escenario está determinado por valores estimados dados por las variables alteradas para cada situación. Normalmente se definen tres escenarios: optimista, normal y pesimista; cada escenario nos permite examinar los resultados cuando alteramos las variables, midiendo sus cambios tanto porcentual como relativamente, y de esa forma encontrar un valor de empresa, contemplando diferentes posibilidades del comportamiento económico del ente.

Tabla 14. Valor de Empresa

\begin{tabular}{lc}
\hline \multicolumn{2}{c}{ Valor Empresa } \\
\hline Valor Esperado & $\$ 5.948 .801 .338$ \\
\hline Varinza & $\$ 2.028 .286 .441 .203 .850 .000,00$ \\
\hline Desv est & $\$ 1.424 .179 .216,67$ \\
\hline Coef var & 0,2394060813 \\
\hline Precio Base de Negociacion & $\$ 4.524 .622 .121$ \\
\hline Precio Techo Negociacion & $\$ 7.372 .980 .554$ \\
\hline
\end{tabular}

Nota: Elaboración propia, Resultado del Valor de Empresa con base a los escenarios previamente planteados respecto a la probabilidad de ocurrencia para obtener el valor esperado, al valor obtenido se le descuenta la desviación estándar para obtener el precio base de negociación o se le agrega dicha desviación para obtener el precio techo de negociación.

De acuerdo a los resultados obtenidos, se tiene un valor de empresa esperado de $\$ 5.948 .801 .338$, el cual es el resultante de las variables aplicadas 
a cada escenario; no obstante el valor que más se acerca a la realidad de la compañía cubriendo el grado de incertidumbre de desempeño es de $\$ 4.524 .622 .121$, por lo tanto, frente a una negociación de la empresa los socios no deben aceptar un valor por debajo del precio base expresado previamente, si el valor ofertado a los socios es igual o superior a dicho valor, la negociación sería asertiva.

El valor base de negociación resultado de la valoración de la empresa estudio de caso nos muestra una cifra razonable con respecto al valor en libros del ente económico, y adicionalmente deja ver su valor por encima de este reflejando la capacidad de la empresa para generar valor y maximizar sus utilidades el cual es el principal objetivo financiero de cualquier proyecto.

\section{Conclusiones}

A partir del diagnóstico financiero, las proyecciones y la determinación del valor de empresa, aplicados a la empresa objeto de estudio se puede concluir:

El mayor generador de valor de la empresa son las ventas incentivadas por la calidad e innovación en el servicio, no obstante el mayor destructor de valor deriva de las fluctuaciones de los costos inherentes a la operación y que no son controlables.

Respecto al diagnóstico financiero, se evidencia cómo influye la caída del mercado y el incremento de los costos en el ejercicio de la empresa.

Los indicadores de liquidez, muestran una empresa estable, capaz de saldar sus pasivos a corto plazo y enfrentar contingencias o incertidumbres que se presenten en desarrollo de su actividad.

En cuanto al diagnóstico basado en los indicadores de endeudamiento muestra que la estructura de la empresa esta apalancada en un alto grado, pero con la solvencia para mantener el curso de sus operaciones.

Los indicadores de rentabilidad resultado del diagnóstico financiero de la empresa muestran su capacidad de generar valor y maximizar sus utilidades; no obstante la empresa debe cuidar sus estrategias de operación para que las recientes variaciones en los índices de costos no afecten su rentabilidad.

El índice de quiebra aplicado a la empresa muestra una fortaleza financiera por parte de su estructura financiera; no obstante habría que contar con un análisis más a fondo de la clasificación de sus activos y pasivos; donde se evidencie la generación de valor a la empresa.

El ejercicio de valoración de la empresa permitió medir su valor desde tres ópticas diferentes, de una forma dinámica y teniendo en cuenta su generación de valor; cumpliendo el objetivo de establecer el valor de la empresa.

Con base a los resultados obtenidos en el valor de empresa, es correcto afirmar que la empresa cuenta con una estabilidad que le permite generar un valor positivo pese a las fluctuaciones e inferencias del mercado, y el incremento de los costos.

Los socios de la empresa de estudio pueden negociar satisfactoriamente la empresa si el valor ofertado es igual o superior a $\$ 4.524 .622 .121$, el cual fue el resultado obtenido como el precio base de negociación en la estimación del valor de la empresa.

El valor base de negociación obtenido bajo el estudio de caso de la empresa es un valor positivo, en la medida en que cubre el valor en libros de la empresa, el cual es estimado por el valor de sus activos, y muestra un incremento de su valor estimando la generación de valor de la estructura financiera de la empresa. 


\section{Referencias}

Alcántara. (2013). Indicadores Financieros. Recuperado de https://www.uaeh.edu. $\mathrm{mx} /$ docencia/P_Presentaciones/Sahagun/Contaduria/Financas/indicacores.pdf

Bonilla, F. (2010). El Valor Económico Agregado (EVA) en el valor del negocio. Revista Nacional de Administración, 1 (1): 55-70. Recuperado de https:// dialnet.unirioja.es/descarga/articulo/3698505.pdf

Crivellini, J. (2010). Historia de las finanzas [en línea]. Recuperado de http:/ / finanbolsa.com/2010/02/09/historia-de-lasfinanzas/

Dussan, C. (2011). ¿Qué es el análisis Horizontal? Recuperado de http://kdussananalisis.blogspot.com.co/2011/12/que-esel-analisis-horizontal.html

García, S. (2003). Valoración de Empresas, Gerencia del Valor y EVA. Cali, Colombia: Prensa Moderna Impresores S.A.

Hevia, C. (2012). Análisis Vertical. Recuperado de https://es.slideshare.net/ krmen01/anlisis-vertical-14580102
Ortiz, J. (2015). La Valoración de Empresa. Semestre Económico, 4 (8), 1-13. Recuperado de https://revistas.udem.edu. co/index.php/economico/article/ view/1394

Tham, J. \& Vélez, I. (2012). Más Allá de Las Proyecciones: El valor Terminal Tópicos. En Valoración de Activos no financieros. Recuperado de https://papers. ssrn.com/sol3/papers.cfm?abstract_ id $=1992634$

Velásquez, R. (2015). Coste de la Deuda (Kd). Recuperado de http://economipedia. com/definiciones/coste-de-la-deudakd.html

Vélez, I. (2001). Decisiones de inversión. Enfocado a la valoración de empresas. Recuperado de http://www.javeriana.edu.co/decisiones/libro_on_line/Tabla.pdf

Vidarte, J. (2009). El flujo de caja descontado como la mejor metodología en la determinación del valor de una empresa. Revista Gestión \& Desarrollo, 6 (2), 103-120. Recuperado de https:/ /www. usbcali.edu.co/sites/default/files/flujodecaja_josevidarte.pdf 